\title{
Síndrome cólica em equinos de uso militar: análise multivariável de fatores de risco
}

\author{
Colic syndrome in military horses: risk factors multivariable analysis \\ Paula Vieira Evans Hossell Laranjeira ${ }^{\mathrm{I}}{ }^{*}$ Fernando Queiroz de Almeida ${ }^{\mathrm{I}}$ \\ Marco Aurélio Ferreira Lopes ${ }^{\mathrm{II}}$ Maria Júlia Salim Pereira ${ }^{\mathrm{I}}$
}

\section{RESUMO}

\begin{abstract}
O objetivo deste estudo foi identificar os fatores associados a episódios de cólica em equinos de unidades militares no Estado do Rio de Janeiro: Regimento Escola de Cavalaria, Academia Militar das Agulhas Negras e Esquadrão Escola de Cavalaria da Polícia Militar. Para tanto, foi conduzido um estudo de caso controle aninhado em uma coorte. Um total de 770 equinos foi estudado, com 362 (47,0\%) equinos classificados como casos e 408 (53,0\%) classificados como controles. A análise multivariável de regressão logística foi utilizada para identificar os fatores preditivos da cólica, mostrando que o sistema de criação dos equinos em confinamento $(O R=3,62 ; 95 \%$ IC 2,57-5,10); o consumo de grãos superior a $6 \mathrm{~kg} \mathrm{dia}^{-1}(O R=2,64 ; 95 \%$ IC 1,54-4,50); е a idade dos equinos, com maior possibilidade de ocorrência da cólica nos animais com idade superior a 16 anos $(O R=2,08$; 95\% IC 1,18-3,67), foram fatores de risco significativamente associados à cólica, após ajustamento para outras variáveis.
\end{abstract}

Palavras-chave: abdômen agudo, epidemiologia, regressão logística, fatores de risco.

\section{ABSTRACT}

This research aimed to identify colic associated factors in horses of military units in Rio de Janeiro State: Regimento Escola de Cavalaria, Academia Militar das Agulhas Negras and Esquadrão Escola de Cavalaria. A case control study nestled in one coorte was conducted. A total of 770 horses had been studied, with 362 (47.0\%) horses classified as cases and 408 (53.0\%) as control. Multivariable analysis of logistic regression was used to identify colic predictive factors, showing that horses confined system creation $(O R=3.62 ; 95 \%$ CI 2.57-5.10), concentrate intake above $6 \mathrm{~kg} \mathrm{day}^{-1}(\mathrm{OR}=2.64$; 95\% CI 1.54-4.50) and equine age, with higher possibility of colic in horses above 16 years $(O R=2.08$; 95\% CI 1.18-3.67), had been risk factors significantly associated with colic, after adjustment for other variables.

Key words: acute abdomen, epidemiology, logistic regression, risk factors.

\section{INTRODUÇÃO}

A síndrome cólica, definida como uma dor abdominal dos equinos, é um dos mais importantes problemas de saúde equina, demandando atendimento veterinário (TRAUB-DARGATZ et al., 2001). Vários fatores são apontados como fatores de risco para cólica e, dentre eles estão, mudanças no tipo, na quantidade e na qualidade do alimento (GONÇALVES et al., 2002), idade (COHEN; PELOSO, 1996; MEHDI; MOHAMMAD, 2006; KANEENE et al., 1997; TINKER et al., 1997a,b), raça (MEHDI; MOHAMMAD, 2006), atividade física (TRAUB-DARGATZ et al.,2001); restrição de acesso ao pasto (HUDSON et al., 2001) e histórico de cólicas anteriores e de cirurgias abdominais (COHEN et al., 1995).

No entanto, ainda há muitas controvérsias quanto à etiologia da cólica que podem ser explicadas, em parte, devido às características da população estudada, ao desenho do estudo e às técnicas de análise. Portanto, estudos devem ser desenvolvidos em diversos contextos e com o emprego de técnicas de análise multivariável para se identificar a contribuição relativa de cada fator de risco, devido a sua natureza multifatorial (KATZ, 2006).

\footnotetext{
Instituto de Veterinária, Universidade Federal Rural do Rio de Janeiro (UFRRJ), Seropédica, RJ, Brasil. *Endereço para correspondência: Rua Comandante Rubens Silva, 62/402, 22745-282, Jacarepaguá, Rio de Janeiro, RJ, Brasil. E-mail: phossell@gmail.com.

IICentro de Ciências Biológicas e da Saúde, Universidade Federal de Viçosa (UFV), Viçosa, MG, Brasil.
} 
Diferenças significativas na incidência de síndrome cólica, em quartéis de uma mesma Instituição Militar do Estado do Rio de Janeiro, foram observadas em uma análise bivariada (LARANJEIRA, 2007), indicando a necessidade de serem analisados os fatores envolvidos nesse risco diferenciado.

No Brasil, apesar da grande relevância da síndrome cólica, não há estudos que tenham empregado uma abordagem multivariável para o estudo do tema. Assim, este estudo tem como objetivo identificar os fatores de risco para síndrome cólica em equinos de uso militar, no Estado do Rio de Janeiro, pelo emprego de análise multivariável, utilizando-se a técnica de análise de regressão logística.

\section{MATERIAL E MÉTODOS}

O estudo foi realizado com base nas informações obtidas de equinos, no período de janeiro de 2003 a dezembro de 2004, pertencentes ao Regimento Escola de Cavalaria (REsC), situado na Vila Militar, em Deodoro, Rio de Janeiro, da Academia Militar das Agulhas Negras (AMAN), situada na cidade de Resende, ambos quartéis do Exército Brasileiro, e do Esquadrão Escola de Cavalaria da Polícia Militar do Estado do Rio de Janeiro (EEC), localizado em Sulacap, Rio de Janeiro, totalizando 770 equinos, sendo 435 pertencentes ao REsC, 261 pertencentes à AMAN e 74 pertencentes ao EEC.

O desenho adotado neste estudo foi do tipo caso-controle aninhado em uma coorte (LARANJEIRA, 2007), o que minimizou a presença de viés de recordação, com a vantagem de não haver perda de informação. Foram considerados casos de cólica todo episódio em que o equino apresentou, pelo menos, um episódio de cólica, caracterizado por sinais de dores abdominais, e que tenha sido atendido pela Seção Veterinária das unidades militares durante o período de observação. Foram considerados casos controles todos os equinos que não apresentaram episódio de cólica. Ao todo, foram analisados $362(47,0 \%)$ equinos casos e 408 (53,0\%) controles.

As exposições (variáveis independentes) analisadas foram: unidade militar: REsC, AMAN e EEC; faixa etária: menor de quatro anos, entre cinco e 15 anos e maior de 16 anos; sexo: masculino e feminino; raça: de raça e mestiço; sistema de criação: estabulado e semiestabulado; fornecimento de volumoso (quantidade por dia): quantidade restrita com feno e/ ou verde na baia e à vontade, com acesso ao pasto e feno oferecido na baia; grãos (incluindo ração concentrada, aveia e linhaça): $4 \mathrm{~kg}$ e $6 \mathrm{~kg}$ ou mais; suplemento mineral-vitamínico: recebia e não recebia; tamanho das baias: $\leq 7,2 \mathrm{~m}^{2} \mathrm{e}>7,2 \mathrm{~m}^{2}$; vício: com vício e sem vício; atividade física: esportiva (Equitação, Adestramento, Concurso Completo de Equitação (CCE), Pólo, Iniciação Desportiva e Instrução de Saltadores), militar (Instrução Militar e Policiamento Montado) e leve (Equoterapia, Escola de Equitação e Lazer) e a variável desfecho, caso de cólica (sim/não).

As variáveis referentes ao sistema de criação e volumoso representaram as mesmas unidades de observação, pois todos os equinos mantidos em sistema semiestabulado possuíam acesso livre ao volumoso no período em que permaneciam livres no pasto e alguns ainda recebiam um complemento de volumoso por meio de feno oferecido na baia. Os equinos estabulados apenas recebiam volumoso na baia, feno e/ou verde, em quantidade limitada por dia. Dessa forma, foi somente considerada a variável sistema de criação.

Um banco de dados montado no programa Microsoft Office Excel 10.0 e elaborado no estudo original, no qual foram introduzidos os dados referentes aos equinos dos plantéis das três unidades militares, foi utilizado. As análises estatísticas foram realizadas com auxílio do programa Statistical Package for the Social Sciences (SPSS) para Windows, versão 13.0.

Com base na variável resposta, que, nesse caso, é dicotômica, foi escolhido o tipo de modelo multivariável para análise, conforme KATZ (2006). Assim, o modelo de análise de regressão logística foi utilizado para conhecer os fatores preditivos da cólica.

O método simultâneo foi escolhido como método de seleção de variáveis para entrar no modelo de regressão logística, incluindo-se todas as variáveis $(\mathrm{n}=10)$ para o primeiro passo. A partir do modelo preliminar, foram escolhidas para a construção do modelo de predição da ocorrência da síndrome cólica as variáveis com valores de $\mathrm{P}=0,20$ e que apresentam relevância clínica ou que, a partir dos resultados disponíveis na literatura, mostram-se potencialmente associadas ao desfecho. A análise multivariável de regressão logística foi realizada pelo método Enter, e o modelo final foi avaliado pela análise da razão de verossimilhança e pelo teste de Hosmer e Lemeshow. No modelo final, permaneceram aquelas variáveis que explicam a ocorrência de síndrome cólica com intervalo de confiança de $95 \%$.

Após a determinação do modelo final, procedeu-se à avaliação das razões de chances ajustadas e seus respectivos intervalos de confiança, bem como da significância do teste de Wald para cada variável. 


\section{RESULTADOS E DISCUSSÃO}

Na tabela 1, é apresentado o modelo preliminar. Apesar de a variável unidade militar ter sido incluída na análise, foi excluída, automaticamente, quando gerado o modelo preliminar. As variáveis sistema de criação, grãos, faixa etária e baia atenderam ao critério de inclusão $(\mathrm{P} \leq 0,20)$ e foram selecionadas para entrar no modelo final. Ainda que a variável baia tenha sido estatisticamente significativa, esta foi excluída quando foi gerado o modelo logístico final, já que não se ajustava adequadamente no modelo.

Na tabela 2, é apresentado o modelo final de regressão logística, considerando as variáveis com relevância estatística e clínica. $\mathrm{O}$ manejo dos equinos estabulados, com limitação do acesso ao volumoso, foi a variável que mostrou a mais forte associação com a cólica, sendo considerado um fator de risco importante para a incidência de síndrome cólica nos equinos militares estudados, pois foi observado que os animais que recebiam uma quantidade restrita de volumoso, por meio de verde e feno, apenas na baia e em horários determinados, possuem a chance 3,62 vezes maior de apresentarem cólica, o que também foi observado por HUDSON et al. (2001). A quantidade de volumoso ingerida ao longo do dia influi de forma efetiva no funcionamento do trato gastrintestinal dos equinos, e os equinos alimentados apenas com pastagem são menos acometidos por cólica (TINKER et al., 1997b; COHEN et al., 1999). Portanto, a restrição ao alimento volumoso pode propiciar a ocorrência de cólica.

A pastagem é o alimento natural dos equinos há milhões de anos, e o equino possui um sistema digestivo adaptado anatômica e fisiologicamente para digerir os alimentos volumosos e suprir as exigências nutricionais. Porém, a estabulação e os esportes equestres que aumentam as exigências nutricionais implicam que sejam fornecidas dietas muitas vezes incompatíveis com a capacidade digestiva e metabólica (HINTZ, 2005). Portanto, buscando uma redução na incidência de cólica nos equinos das unidades militares analisadas, maior atenção deve ser dada à qualidade $\mathrm{e}$ quantidade do volumoso oferecido aos animais, pois há redução na incidência de cólica quando os equinos têm acesso livre ao volumoso (REEVES et al., 1996).

Os resultados deste estudo estão de acordo com GONÇALVES et al. (2002), os quais relacionaram o manejo alimentar, o tipo, a composição e a quantidade dos alimentos como principais fatores que afetam a ocorrência de cólica. A forragem também pode ser fator de risco para ocorrência de cólica se for de baixa digestibilidade e com fibras de baixa qualidade, sendo associada com a ocorrência de compactações (WHITE \& DABAREINER, 1997; LITTLE \& BLIKSLAGER, 2002).

Deve-se levar em consideração que o tipo de alimentação deve ser adequado para cada animal, o que varia de acordo com o tipo de atividade física a que é submetido. Logo, as dietas dos animais deveriam

Tabela 1 - Análise de regressão logística dos fatores de risco para síndrome cólica em equinos de três unidades militares, no Estado do Rio de Janeiro, no período 2003-2004 - modelo preliminar.

\begin{tabular}{|c|c|c|c|c|c|c|}
\hline \multirow{2}{*}{ Variáveis } & \multirow{2}{*}{$\mathrm{B}$} & \multirow{2}{*}{$\chi^{2}$ Wald } & \multirow{2}{*}{ p-valor } & \multirow{2}{*}{$\begin{array}{c}\text { Exp (B) Odds } \\
\text { Ratio }\end{array}$} & \multicolumn{2}{|c|}{----Intervalo de Confiança 95,0\%---- } \\
\hline & & & & & Inferior & Superior \\
\hline Sistema de criação (estabulado) & 2,155 & 64,333 & 0,000 & 8,629 & 5,096 & 14,610 \\
\hline Grãos $\left(\geq 6 \mathrm{~kg} \mathrm{dia}^{-1}\right)$ & 0,477 & 1,651 & 0,199 & 1,611 & 0,778 & 3,334 \\
\hline Suplemento mineral-vitamínico & $-0,081$ & 0,050 & 0,823 & 0,922 & 0,453 & 1,876 \\
\hline \multicolumn{7}{|l|}{ Faixa etária (anos) } \\
\hline$\leq 4$ & & 4,858 & 0,088 & & & \\
\hline $5-15$ & 0,351 & 2,825 & 0,093 & 1,421 & 0,943 & 2,141 \\
\hline$\geq 16$ & 0,644 & 4,435 & 0,035 & 1,904 & 1,046 & 3,466 \\
\hline Baia $\left(=7,2 \mathrm{~m}^{2}\right)$ & $-0,952$ & 15,243 & 0,000 & 0,386 & 0,239 & 0,623 \\
\hline Sexo (masculino) & 0,053 & 0,100 & 0,751 & 1,055 & 0,758 & 1,468 \\
\hline Vício & $-0,180$ & 0,122 & 0,727 & 0,836 & 0,305 & 2,288 \\
\hline Raça & $-0,260$ & 1,568 & 0,210 & 0,771 & 0,513 & 1,158 \\
\hline \multicolumn{7}{|l|}{ Atividade } \\
\hline Leve & & 11,325 & 0,003 & & & \\
\hline Esportiva & $-0,181$ & 0,221 & 0,639 & 0,835 & 0,393 & 1,774 \\
\hline Militar & 0,605 & 2,100 & 0,147 & 1,830 & 0,808 & 4,146 \\
\hline Constante & $-1,028$ & 3,277 & 0,070 & 0,358 & & \\
\hline
\end{tabular}

Ciência Rural, v.39, n.6, set, 2009. 
Tabela 2 - Análise de regressão logística dos fatores de risco para síndrome cólica em equinos de três unidades militares, no Estado do Rio de Janeiro, no período 2003-2004 - modelo final.

\begin{tabular}{|c|c|c|c|c|c|c|}
\hline \multirow{2}{*}{ Variáveis } & \multirow{2}{*}{$\mathrm{B}$} & \multirow{2}{*}{$\chi^{2}$ Wald } & \multirow{2}{*}{ p-valor } & \multirow{2}{*}{$\begin{array}{c}\text { Exp (B) Odds } \\
\text { Ratio }\end{array}$} & \multicolumn{2}{|c|}{----Intervalo de Confiança 95,0\%---- } \\
\hline & & & & & Inferior & Superior \\
\hline Sistema de criação (estabulado) & 1,287 & 54,157 & 0,000 & 3,622 & 2,571 & 5,102 \\
\hline Grãos $\left(\geq 6 \mathrm{~kg} \mathrm{dia}^{-1}\right)$ & 0,969 & 12,598 & 0,000 & 2,635 & 1,543 & 4,500 \\
\hline \multicolumn{7}{|l|}{ Faixa etária (anos) } \\
\hline$\leq 4$ & & 8,066 & 0,018 & & & \\
\hline $5-15$ & 0,478 & 5,903 & 0,015 & 1,612 & 1,097 & 2,371 \\
\hline$\geq 16$ & 0,734 & 6,463 & 0,011 & 2,083 & 1,183 & 3,668 \\
\hline Constante & $-1,795$ & 34,802 & 0,000 & 0,166 & & \\
\hline
\end{tabular}

ser adaptadas para as exigências nutricionais de acordo com o nível e a intensidade de utilização de cada equino individualmente, pois o excesso e a falta de atividade física podem estar relacionados com a cólica, como foi observado por WHITE (1995), apesar de o autor não ter usado análises multivariáveis. Os equinos, em atividades de tração, equitação, corrida e salto, realizam trabalho muscular intenso e, nos diversos grupos de atividades esportivas, a intensidade e o tempo de treinamento são diferentes. Cada tipo de trabalho exige alterações na formulação e no fracionamento da dieta (MEYER, 1995).

Os equinos que ingerem seis quilogramas ou mais de grãos por dia possuem 2,64 vezes mais chances de apresentarem cólica quando comparados com aqueles que ingerem quatro quilogramas de grãos por dia. A quantidade de grãos ingerida é um fator de risco importante para a ocorrência de cólica, devido à quantidade de carboidratos hidrolisáveis presentes na dieta (WHITE, 1995). Isso também foi observado em estudos realizados por TINKER et al. (1997b), os quais observaram risco maior de cólica para os equinos que ingeriam maiores quantidades de concentrado.

As possíveis mudanças na dieta devem ser graduais para que ocorra uma adaptação do organismo do equino à nova alimentação. Mudanças súbitas na dieta podem causar indigestão e cólica (COHEN et al., 1995, 1999; REEVES et al., 1996). Portanto, deve-se sempre atentar para a qualidade e quantidade de concentrado oferecido ao equino, pois o aumento da quantidade de grãos oferecidos nas refeições pode elevar o risco de ocorrência de cólica (CLARKE et al., 1990; REEVES et al., 1996; TINKER et al., 1997b; JULLIAND et al., 2001).

Os resultados do presente estudo indicam que o fornecimento de uma alimentação controlada e adaptada ao equino e a possibilidade do animal exercitar-se diariamente de maneira adequada podem ser formas seguras para proteger ou minimizar o risco de ocorrência da síndrome cólica.
A idade dos equinos também foi identificada como um fator de risco para a ocorrência de síndrome cólica. Os equinos com idade entre cinco e 15 anos possuem 1,61vezes mais chance de ter cólica em relação aqueles com idade até quatro anos. Por outro lado, os equinos com idade igual ou superior a 16 anos possuem 2,08 vezes mais chance de ter cólica em relação aos equinos com idade inferior a quatro anos. Portanto, o risco de cólica aumenta com o aumento da idade do equino. Os equinos, na faixa de idade entre cinco e quinze anos, estão em ampla atividade física e os equinos com mais idade tendem a apresentar mais cólica, pois podem possuir outras alterações em seu trato gastrintestinal que podem o predispor a novo episódio de cólica. Também os equinos mais velhos tendem a possuir algumas alterações na arcada dentária que podem prejudicar a adequada apreensão, mastigação e ingestão dos alimentos (PAGLIOSA et al., 2006), favorecendo a ocorrência de alterações que levam ao quadro de síndrome cólica, pois a mastigação deficiente leva à trituração insuficiente dos alimentos e à diminuição da produção de saliva, o que pode afetar a digestibilidade dos alimentos e o trânsito intestinal (MEYER, 1995).

Segundo THOEFNER et al. (2001), a idade, juntamente com fatores como a endotoxemia, o timpanismo cecal e a raça, explicam $51 \%$ da variabilidade dos dados. A influência da idade também foi observada em outros estudos (TENNANT et al., 1972; SEMBRAT, 1975; MORRIS et al., 1989; REID et al., 1995; WHITE, 1995; COHEN \& PELOSO, 1996; TINKER etal., 1997a,b; MEDHI \& MOHAMMAD, 2006). Porém, nem todos esses autores usaram um modelo de análise multivariável visando ao controle da idade por outros fatores. Neste estudo, as variáveis baia, vício e tamanho de baia não contribuíram para explicar a incidência de cólica nas unidades militares.

O modelo mostrou-se adequado para predição da ocorrência de cólica em equinos das três 
unidades militares, tendo em vista que o resultado do teste de Hosmer e Lemeshow $(\mathrm{P}=0,896)$ indica que não houve diferenças entre os valores estimados para a ocorrência da síndrome cólica e os valores reais.

À análise do p-valor $(\mathrm{P}<0,01)$, observou-se que o conjunto de variáveis incluídas no modelo contribui significativamente para predição do desfecho, ou seja, da ocorrência de síndrome cólica.

\section{CONCLUSÃO}

Os resultados deste estudo permitiram identificar como fatores de risco para a síndrome cólica nas unidades militares, por ordem decrescente de significância estatística, a estabulação e a restrição de volumoso, a grande quantidade de grãos ingeridos por dia, com os equinos que ingerem mais de seis quilogramas de grãos por dia em maior risco, em comparação com a menor ingestão, e a faixa etária, sendo a faixa de idade de 16 ou mais anos a de maior risco, seguida da faixa entre cinco e 15 anos.

\section{AGRADECIMENTOS E APRESENTAÇÕES}

Este estudo é parte da tese do primeiro autor, realizada no Curso de Pós-graduação em Ciências Veterinárias da Universidade Federal Rural do Rio de Janeiro (UFRRJ) e financiada pelo Conselho Nacional de Desenvolvimento Científico e Tecnológico $(\mathrm{CNPq})$ /Fundação de amparo à Pesquisa do Estado do Rio de Janeiro (FAPERJ).

\section{REFERÊNCIAS}

CLARKE, L.L. et al. Feeding and digestive problems in horses: physiologic responses to a concentrated meal. Veterinary Clinics of North America: Equine Practice, v.6, n.2, p.433450, 1990.

COHEN, N.D.; PELOSO, J.G. Risk factors for history of previous colic and for chronic, intermittent colic in a population of horses. Journal of American Veterinary Medical Association, v.208, n.5, p.697-703, 1996.

COHEN, N.D. et al. Dietary and other management factors associated with colic in horses. Journal of American Veterinary Medical Association, v.215, n.1, p.53-60, 1999.

COHEN, N.D. et al. Case-control study of the association between various management factors and development of colic in horses. Journal of American Veterinary Medical Association, v.206, n.5, p.667-673, 1995.

GONÇALVES, S. et al. Risk factors associated with colic in horses. Veterinary Research, v.33, n.6, p.641-652. 2002. Disponível em: <http://www.vetres.org/ index.php?option $=$ article\&access $=$ standard\&Itemid $=129 \&$ url $=/$ articles/vetres/pdf/2002/06/01.pdf $>$. Acesso em: 13/01/ 2009. Doi: $10.1051 /$ vetres:2002044
HINTZ, H.F. Evaluate the entire equine diet. Journal of Equine Veterinary Science, v.25, n.12, p.540-541. 2005. Disponível em: <http://www.sciencedirect.com/ science? ob=ArticleURL\& udi=B75GX-4HW3MP3D\&_user $=687350 \&$ rdoc $=1 \&$ fmt $=$ \&_orig $=$ search \&_sort $=\mathrm{d} \&$ view $=\mathrm{c} \&$ acct $=\mathrm{C} 000037881 \&$ version $=1 \&$ urlVersion $=0 \&$ userid $=687350 \& \mathrm{md} 5=19705 \mathrm{dc} 8 \mathrm{ff} 035 \mathrm{~d} 86 \mathrm{dc} 585 \mathrm{~d} 2261 \mathrm{c} 39 \mathrm{de} 6>$ Acesso em: 27/04/2009. Doi:10.1016/j.jevs.2005.11.009.

HUDSON, J.M. et al. Feeding practices associated with colic in horses. Journal of American Veterinary Medical Association, v.219, n.10, p.1419-1425, 2001.

JULLIAND, V. et al Feeding and microbial disorders in horses: effects of three hay, grain rations on microbial profile and activities. Journal of Equine Veterinary Science, v.21, n.11, p.543-546, 2001. Disponível em: <http:// linkinghub.elsevier.com/retrieve/pii/S0737080601701591>. Acesso em: 27/04/2009. Doi:10.1016/S0737-0806(01)70159-1

KANEENE, J.B. et al. Risk factors for colic in Michigan (USA) equine population. Preventive Veterinary Medicine, v.30, n.1, p.23-36, 1997.

KATZ, M.H. Multivariable analysis. A pratical guide for clinicians. 2.ed. Cambridge: Cambridge University, 2006. 203 p.

LARANJEIRA, P.V.E.H. Fatores de risco para síndrome cólica em eqüinos de uso militar no Estado do Rio de Janeiro. 2007. 67f. Tese (Doutorado em Ciências Veterinárias) - Curso de Pós-graduação em Ciências Veterinárias, Universidade Federal Rural do Rio de Janeiro.

LITTLE, D.; BLIKSLAGER, A.T. Factors associated with development of ileal impaction in horses with surgical colic: 78 cases (1986-2000). Equine Veterinary Journal, v.34, n.5, p.464-468, 2002 .

MEHDI, S.; MOHAMMAD, V. A farm-based prospective study of equine colic incidence and associated risk factors. Journal of Equine Veterinary Science, v.26, n.4, p.171174, 2006. Disponível em: <http://www.sciencedirect.com/ science?_ob=MImg\&_imagekey=B75GX-4JS81 T3-C$1 \&$ cdi $=13106 \&$ user $=687350 \&$ orig=browse \& cover Date $=04 \% 2 F 30 \% 2 F 2006 \&$ sk $=999739995 \&$ view $=c \& w c h p=d \bar{G} L b V t b-$ zSkzS\&md5=ae 5 fe 4 c 1 f9efdec 24 bb 442 dae 9 c 5 f9 2 c \& ie $=/$ sdarticle.pdf $>$. Acesso em: 1 dez. 2008. Doi: 10.1016/ j.jevs.2006.02.008

MEYER, H. Alimentação de cavalos. São Paulo: Varela, 1995. 303p.

MORRIS, D.D. et al. Comparison of age, sex, breed, history and management in 229 horses with colic. Equine Veterinary Journal, v.7, (Suppl), p.129-132, 1989.

PAGLIOSA, G.M. et al. Influência das pontas excessivas de esmalte dentário na digestibilidade e nutrientes de dietas de eqüinos. Arquivo Brasileiro de Medicina Veterinária e Zootecnia, v. 58, n. 1, p. 94-98, 2006.

REEVES, M.J. et al. Risk factors for equine acute abdominal disease (colic): Results from a multi-center case-control study. Preventive Veterinary Medicine, v.26, n. 3, 
p.285-301, 1996. Disponível em: <http://www.vetres.org/ index.php?option $=$ article \&access $=$ standard \&Itemid $=129 \&$ url $=/$ articles/vetres/pdf/2002/06/01.pdf.> Acesso em: 28 nov. 2008. Doi: 10.1051/vetres:2002044

REID, S.W.J. et al. Epidemiological risk factors associated with a diagnosis of clinical cyathostomiasis in the horse. Equine Veterinary Journal, v.27, n.2, p.127-130, 1995.

SEMBRAT, R.F. The acute abdomen in the horse epidemiologic considerations. Archive Official of Journal of the American College of Veterinary Surgeons, v.4, p.34-39, 1975.

TENNANT, B. et al. Observations on the causes and incidence of acute intestinal obstruction in the horse. In: ANNUAL CONVENTION OF THE AMERICAN ASSOCIATION OF EQUINE PRACTITIONERS, 18., 1972, San Francisco. Proceedings... California: AAEP, 1972. p.251-257.

THOEFNER, M.B. et al. Factor analysis of the interrelationships between clinical variables in horses with colic. Preventive Veterinary Medicine, v.48, n.3, p.201-214, 2001.
TINKER, M.K. et al. Prospective study of equine colic incidence and mortality. Equine Veterinary Journal, v.29, n.6, p.448453, 1997a.

TINKER, M.K. et al. Prospective study of equine colic risk factors. Equine Veterinary Journal, v.29, n.6, p.454-458, $1997 b$.

TRAUB-DARGATZ, J.L. et al. Estimate of the national incidence of and operation-level risk factors for colic among horses in the United States, spring 1998 to spring 1999. Journal of American Veterinary Medical Association, v.219, n.1, p.67-71, 2001.

WHITE, N.A. Epidemiology of equine colic. In: CICLO INTERNACIONAL DE CÓLICA EQÜINA, 2., 1995, UNESPJaboticabal, SP. Anais... Jaboticabal: FCAV-UNESP, 1995. p. $1-9$.

WHITE, N.A.; DABAREINER, R.M. Treatment of impaction colics. Veterinary Clinics of North America: Equine Practice, v.13, n.2, p.243-259, 1997. 